\title{
Asymptotic analysis of subcritical Hopf-homoclinic bifurcation
}

\author{
John Guckenheimer ${ }^{\mathrm{a}, *}$, Allan R. Willms ${ }^{\mathrm{b}}$ \\ ${ }^{a}$ Mathematics Department and Center for Applied Mathematics, Cornell University, Ithaca, NY 14853, USA \\ ${ }^{\mathrm{b}}$ Biomathematics Research Centre, Department of Mathematics and Statistics, University of Canterbury, \\ Private Bag 4800, Christchurch, New Zealand
}

Received 5 August 1998; received in revised form 2 November 1999; accepted 4 November 1999

Communicated by C.K.R.T. Jones

\begin{abstract}
This paper discusses the mathematical analysis of a codimension two bifurcation determined by the coincidence of a subcritical Hopf bifurcation with a homoclinic orbit of the Hopf equilibrium. Our work is motivated by our previous analysis of a Hodgkin-Huxley neuron model which possesses a subcritical Hopf bifurcation (J. Guckenheimer, R. Harris-Warrick, J. Peck, A. Willms, J. Comput. Neurosci. 4 (1997) 257-277). In this model, the Hopf bifurcation has the additional feature that trajectories beginning near the unstable manifold of the equilibrium point return to pass through a small neighborhood of the equilibrium, that is, the Hopf bifurcation appears to be close to a homoclinic bifurcation as well. This model of the lateral pyloric (LP) cell of the lobster stomatogastric ganglion was analyzed for its ability to explain the phenomenon of spike-frequency adaptation, in which the time intervals between successive spikes grow longer until the cell eventually becomes quiescent. The presence of a subcritical Hopf bifurcation in this model was the one identified mechanism for oscillatory trajectories to increase their period and finally collapse to a non-oscillatory solution. The analysis presented here explains the apparent proximity of homoclinic and Hopf bifurcations. We also develop an asymptotic theory for the scaling properties of the interspike intervals in a singularly perturbed system undergoing subcritical Hopf bifurcation that may be close to a codimension two subcritical Hopf-homoclinic bifurcation. (C) 2000 Elsevier Science B.V. All rights reserved.
\end{abstract}

Keywords: Hopf bifurcation; Dynamic bifurcation; Homoclinic orbit; Spike-frequency adaptation; Nonlinear oscillations

\section{Introduction}

The characterization of generic bifurcations is one of the major achievements of dynamical systems theory. Bifurcation theory provides a substrate for interpreting observed qualitative changes in attractors and other limit sets of dynamical systems. One of the crucial properties of a bifurcation is its codimension, roughly the minimal number of parameters in a family of dynamical systems that contains the bifurcation in a persistent manner. Here,

\footnotetext{
in Research partially supported by the National Science Foundation.

* Corresponding author.

E-mail address: gucken@can.cornell.edu (J. Guckenheimer)
} 


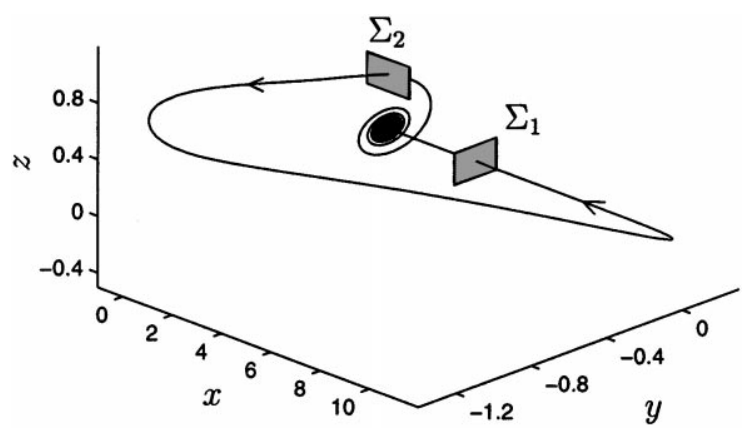

Fig. 1. Flow past the Hopf-homoclinic point.

we investigate a codimension two bifurcation that results from the confluence of Hopf bifurcation and homoclinic bifurcation, each a codimension one bifurcation [6]. We were motivated to examine the questions addressed in this paper by our studies of spike-frequency adaptation in neurons [5]. There are two issues that arose in that work that prompted the more detailed mathematical study here.

In our numerical investigations of a model neuron, we observed subcritical Hopf bifurcations that appeared to be accompanied by trajectories that were nearly homoclinic. Fig. 1 depicts a three-dimensional representation of such a trajectory. We expected that the coincidence of Hopf and homoclinic bifurcations would not take place in generic one parameter families of vector fields, prompting an investigation of the relationship between Hopf and homoclinic bifurcations. The way in which we pursue that topic in this paper is to study the unfoldings of two parameter families in which subcritical Hopf-homoclinic bifurcation occurs in a persistent manner. We demonstrate that, in accord with our expectations, Hopf-homoclinic bifurcation does not occur in generic one parameter families. Nonetheless, trajectories that appear to approximate Hopf-homoclinic bifurcation are readily produced by Hopf bifurcations even when the systems are not close to homoclinic bifurcations in multiparameter families. We develop an explanation for this behavior in this paper. Briefly, it is caused by transverse stability along the stable manifold of an equilibrium point that has a (weakly) unstable manifold created by Hopf bifurcation. Large regions of the phase space are attracted to a neighborhood of the stable manifold and flow along this stable manifold close to the equilibrium point before they are repelled along its unstable manifold. We believe that this dynamical behavior is common [4], but that the role of Hopf bifurcation in creating approximate homoclinic trajectories has not been highlighted previously.

The second issue that we address was more directly motivated by our desire to analyze data from neurons undergoing spike-frequency adaptation. Spiking neurons generate repetitive action potentials, rapid increases of membrane potential that can be interpreted as relaxation oscillations. In spike-frequency adaptation, the firing rate of the action potentials slows and the period of the oscillations increases. In some circumstances, the firing terminates spontaneously, a phenomenon that we call the death of periodicity. In bursting oscillations of neural systems, the death of periodicity occurs repeatedly as the system oscillates between firing and quiescent epochs on a timescale slower than that of the periodic action potentials. The death of periodicity can be modeled with slowly varying dynamical systems in which one set of variables evolves on slower timescales than others. In the singular limit in which the slow timescale is frozen, the slow variables become parameters. Qualitative changes along a trajectory, like the death of periodicity, become bifurcations in the frozen system.

We want to identify the type of bifurcation underlying the death of periodicity of an observed time series. The most prevalent data from neural systems are sampled records of membrane potential. The timing of action potentials is evident in these records and the interspike intervals can be measured with a good degree of precision. Rinzel and Ermentrout [9] observed that different types of bifurcations yield different asymptotic rates for the 
lengthening of interspike intervals. They noted this phenomenon in the context of homoclinic and saddle-node in cycle bifurcations underlying the death of periodicity. We sought to quantify this observation and apply it to data from neurons in the stomatogastric ganglion, when we observed subcritical Hopf bifurcation as an additional mechanism responsible for the death of periodicity. This prompted our investigation of how the periods of long periodic orbits in systems close to subcritical Hopf bifurcation depend upon the distance to the bifurcation. When a system is close to both homoclinic and Hopf bifurcation, as appeared to be the case, we sought to determine parameter regions in which proximity to Hopf bifurcation or to homoclinic bifurcation was more important in determining the periods of long periodic orbits. We sought a uniform asymptotic expression for the length of periodic orbits that are close to codimension two Hopf-homoclinic bifurcations, but we have only obtained partial results that give non-uniform asymptotic expressions in different parameter regimes. The details of these results are complicated, but the predominant conclusion is that a system must be much closer to homoclinic bifurcation than to Hopf bifurcation in order for the homoclinicity to play a substantial role in lengthening the period of the periodic orbit.

We begin this paper with numerical computations from a three-dimensional example which illustrate some of the dynamical phenomena we wish to discuss. Our purpose is twofold. First, we give a geometrical description of the return map to the vicinity of the equilibrium point near the Hopf-homoclinic bifurcation, showing some of its complexity and how it gives rise to chaotic sets and long stable periodic orbits. For a slowly varying system, trajectories will typically track a family of stable periodic orbits until the bifurcation is reached, after which they become quiescent. Second, we develop expressions for the time taken for trajectories to pass through the region of the equilibrium near the Hopf-homoclinic bifurcation. We show that when the system is frozen at the Hopf bifurcation, trajectories passing near the equilibrium point spend an amount of time inversely proportional to the square of the distance, $r_{1}$, to the stable manifold before escaping the region. If the system parameters are frozen at some point before the Hopf bifurcation, the slow spiraling away from the equilibrium point becomes the dominant feature. The travel time is then inversely proportional to the real part of the Hopf eigenvalue, $\mu_{0}$ (positive), but modified by a logarithmic factor which depends on $\mu_{0}$ and $r_{1}$. For frozen systems, the scaling of the spiking period as $1 / \mu_{0}$ is a characteristic feature of this bifurcation. In case of a slowly varying system where the real part of the Hopf eigenvalue, $\mu$, decreases linearly with time, the travel time also depends on $s$, the rate of change of $\mu$ and the time remaining before $\mu$ reaches zero. We develop asymptotic expressions for the travel time relating the slowly varying system to the frozen system and we also present justification for a simple functional form

$$
\frac{f\left(r_{1}\right)}{g\left(r_{1}, s\right)+\mu},
$$

which characterizes the lengthening of the travel time as the bifurcation is slowly approached. We argue that in a generic system passing near a subcritical Hopf-homoclinic bifurcation in this manner, the variation in the distance, $r_{1}$, to the stable manifold is not as significant as the decrease in the real part of the Hopf eigenvalue with time. Setting $\mu=-s t_{2}$, where $t_{2} \leq 0$, the Hopf bifurcation will occur at time zero and the spiking period will show a fractional linear dependence on $\left|t_{2}\right|$. We claim that when the transition to quiescence is still a number of oscillations away, the chief visible characteristic feature of the Hopf-homoclinic bifurcation is this fractional linear dependence of the interspike intervals on the time remaining before the Hopf bifurcation is reached.

In the LP cell model which we analyzed in Guckenheimer et al. [5], we found that the subcritical Hopf bifurcation seems to exist over a fairly wide range of physiologically realistic parameter values and can clearly affect the adaptation in the length of the interspike intervals when the model cell is near this bifurcation and undergoing a transition from an oscillatory mode to a quiescent one. For this case we used the following expression to describe the increasing length of the interspike intervals:

$$
\text { Period }=a+\frac{1}{b-c t}
$$


where, $t \leq 0$, and $a$ represents the (almost) constant amount of time taken for the trajectory to pass through the region far from the equilibrium point and the form of the second term is justified by the fractional linear functional form for the travel time of the trajectory through the region near the equilibrium point which we develop in this paper. In the previous paper we had also identified two other transition mechanisms for which the period of oscillation was unbounded as the transition was approached and developed asymptotic expressions for the lengthening of the interspike intervals in each case. We found that it was possible to distinguish the model mechanism by fitting the generated interspike interval data to each of these expressions. In particular, we found that (2) successfully fit interspike interval data from both the model LP cell simulations and from experiments on the real LP cell.

The coincidence of supercritical Hopf and homoclinic bifurcations has been discussed by Hirsch and Knobloch [7] and Bosch and Simo [2], but the phenomenology of that case is substantially different. The subcritical case, where the equilibrium point gives birth to unstable limit cycles, was first studied by Belyakov [1] in 1974, who showed that the homoclinic orbit of the equilibrium point bifurcates into two 1-pass homoclinic orbits of the nascent unstable limit cycle as the Hopf bifurcation curve is crossed. In 1995, Deng and Sakamoto [3] confirmed and extended this analysis to give a description of the system's behavior in a two-dimensional parameter neighborhood of the Hopf-homoclinic bifurcation. Deng and Sakamoto give a fairly comprehensive discussion of the existence of chaotic and homoclinic trajectories of the subcritical Hopf-homoclinic bifurcation. The scaling properties of the cycles near Hopf-homoclinic orbits are the main subject of this paper. They have not been analyzed previously.

\section{A numerical example}

We first illustrate some of the results we will later establish by analyzing a three-dimensional vector field designed to have a Hopf-homoclinic transition:

$$
\begin{aligned}
& \dot{x}=-x+A\left(y^{2}+z^{2}\right)+B x^{2} \sqrt{\epsilon^{2}+y^{2}+z^{2}} \\
& \dot{y}=y\left(\left(\mu_{0}-s t\right)-x+\left(y^{2}+z^{2}\right)-C\left(y^{2}+z^{2}\right)^{2}\right)-\omega z+D x^{4}, \\
& \dot{z}=z\left(\left(\mu_{0}-s t\right)-x+\left(y^{2}+z^{2}\right)-C\left(y^{2}+z^{2}\right)^{2}\right)+\omega y+E y^{4} .
\end{aligned}
$$

This vector field has an equilibrium point at the origin, in a neighborhood of which it is approximated in polar coordinates by

$$
\begin{aligned}
\frac{\mathrm{d} x}{\mathrm{~d} t} & =-x, \\
\frac{\mathrm{d} r}{\mathrm{~d} t} & =\left(\mu_{0}-s t\right) r-x r+r^{3}, \quad t \leq 0 . \\
\frac{\mathrm{d} \theta}{\mathrm{d} t} & =\omega .
\end{aligned}
$$

(We shall study this system in Section 5.) The eigenvalues at the origin are -1 and $\mu \pm \mathrm{i} \omega$ and the eigenspaces remain on the $x$-axis and the $(y, z)$ coordinate plane. We investigate the system with $s=0$ and $\mu_{0} \geq 0$ and with $s>0$ and $\mu_{0}=0$. When $\mu=\mu_{0}-s t$ is positive, the origin is a saddle with a stable real eigenvalue and a pair of unstable complex eigenvalues. A subcritical Hopf bifurcation occurs when $\mu$ passes through zero. The terms involving $A, B$ and $C$ were added to propel trajectories away from the unstable eigenspace and inject them back toward the stable eigenspace and the terms with $D$ and $E$ are symmetry breaking terms. The values of the upper case constants were chosen so that all other equilibria in the system remained unstable and so that trajectories near 
A

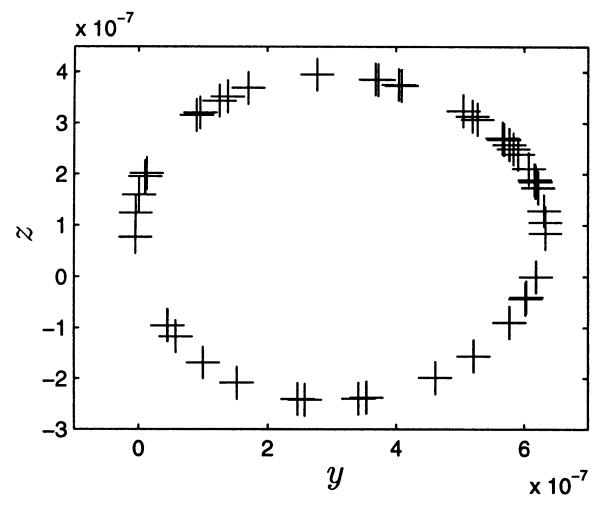

B

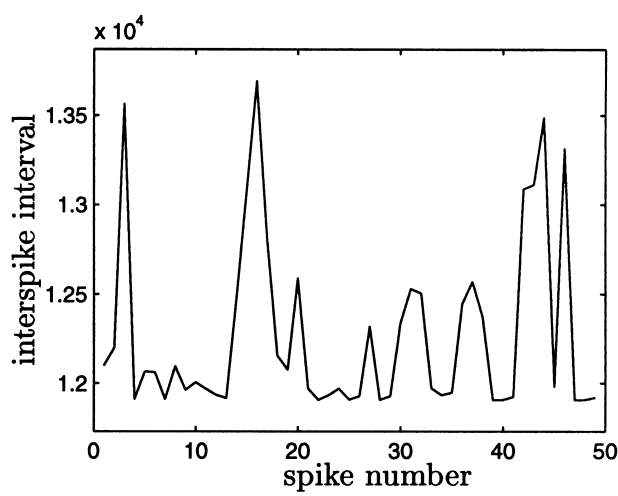

Fig. 2. (A) Intersections with $\Sigma_{1}$ for a near homoclinic trajectory; (B) interspike intervals for the same trajectory.

the unstable manifold of the origin would return close to the origin. To keep the square root term smooth at the origin, $\epsilon$ was chosen as a small non-zero constant.

First, consider the frozen system, $s=0$. There is no apparent analytic procedure for locating homoclinic orbits in this system. We used the following strategy. A segment $\gamma$ of initial conditions lying approximately on the unstable manifold near the origin was chosen and integrated forward until trajectories intersected a cross-section to the stable manifold of the origin. Backwards integration of a trajectory starting near the origin along its stable manifold was used to find the intersection of the cross-section with the stable manifold. When the parameter $D$ is zero, the $x$-axis is invariant for the flow and there are no homoclinic orbits. As $D$ varied, the image of $\gamma$ in the cross-section appeared to cross the intersection of the stable manifold. Using a divide and conquer algorithm, we located parameter values and initial conditions that appear to be close to a homoclinic orbit. The trajectory shown in Fig. 1 is the near homoclinic orbit we identified for this system. The value of $\mu_{0}$ for this trajectory is 0.001 , so that the system is also very close to the Hopf bifurcation curve. Trying to find a homoclinic orbit precisely at the Hopf bifurcation, i.e. $\mu_{0}=0$, is computationally difficult. The non-exponential growth away from the equilibrium point (the term $r^{3}$ in the equation for $\dot{r}$ ) is so small compared to the term $-x r$, that more than 16 digits of precision is required in order to see the slow expansion.

Our theory will predict that homoclinic orbits near a subcritical Hopf bifurcation sit inside chaotic invariant sets. We computed intersections of a long trajectory with a cross-section, $\Sigma_{1}$, for the same parameter values as a system with an approximate homoclinic orbit. Fig. 2A illustrates that these intersections lie close to a curve that seems to form a chaotic attractor. Fig. 2B shows a plot of the interspike intervals for the same trajectory. The interspike intervals within this chaotic attractor are not monotone and vary substantially, which, we shall see, is in accord with the theoretical predictions.

We also computed the interspike intervals of trajectories of various frozen $(s=0)$ systems at differing distances from the Hopf bifurcation (differing values of $\mu_{0}$ ). We observed that the relative variation of return times to a cross-section for each trajectory was much less than in the case where the system was very close to the Hopf-homoclinic transition. As the value of the parameter $\mu_{0}$ approached zero, the return time grew at a rate roughly proportional to $1 / \mu_{0}$, which, corresponds with the bounds on the return times we develop in Section 5.2. Fig. 3 is a $\log -\log$ plot of the computed return times to the cross-section $\Sigma_{1}$ as a function of $\mu_{0}$. The slope of the points is very nearly minus one and the vertical shift on the axis corresponds well to the proportionality factor in the bounds that we will develop ((22) and (23)), given that the the distance to the stable manifold, $r_{1}$, for all of these trajectories is very small, about $10^{-7}$ and where $x_{1}=1$ and $r_{2} \approx 1$. 


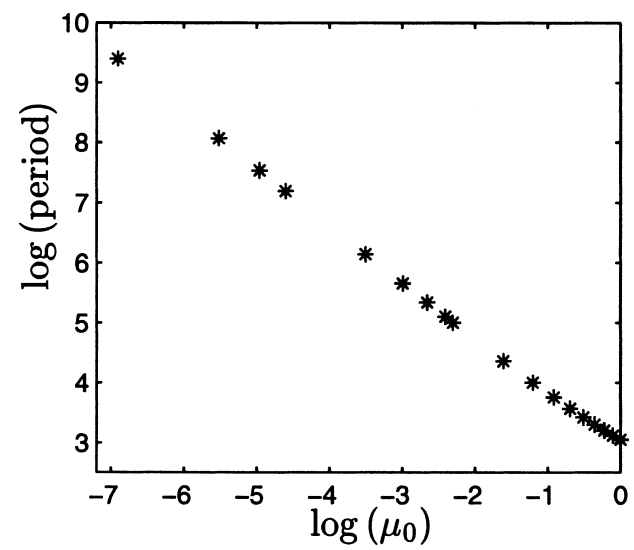

Fig. 3. Log- $\log$ plot of the return time for different values of the Hopf parameter, showing the $1 / \mu_{0}$ dependence.

Finally, we investigated the slowly varying system $\left(s \neq 0, \mu_{0}=0\right)$ as it crossed the Hopf bifurcation near the Hopf-homoclinic point. Fig. 4 shows a typical trajectory with lengthening interspike intervals as the Hopf bifurcation is approached and a transition to quiescence near the Hopf bifurcation $(t=0)$. Fig. 5 shows a plot of the interspike intervals for several such trajectories with different values of $s$. The data points for each of the trajectories, less the final three spikes, were fit to curves of the form

$$
\mathrm{ISI}=a+\frac{b}{c-s t},
$$

where again, $a$ represents the assumed constant amount of time required to traverse the region far from the equilibrium point and the second term corresponds to the simple functional form, (1), which we establish in this paper for the travel time through the region near the equilibrium. Since the value of $r_{1}$ was nearly the same (about $10^{-7}$ ) for all of these trajectories and did not vary substantially with each subsequent pass, the functional form (1) predicts that the parameters $a$ and $b$ should be nearly the same for each curve, while $c$ may show some variability with $s$. Further, in Section 6 we show that the numerator, $b$, should be approximately $\ln \left(r_{2} / r_{1}\right)$. The maximum likelihood estimates of the parameters for these six trajectories are given in Table 1 and verify the above two predictions (taking $r_{2} \approx 1$ ).

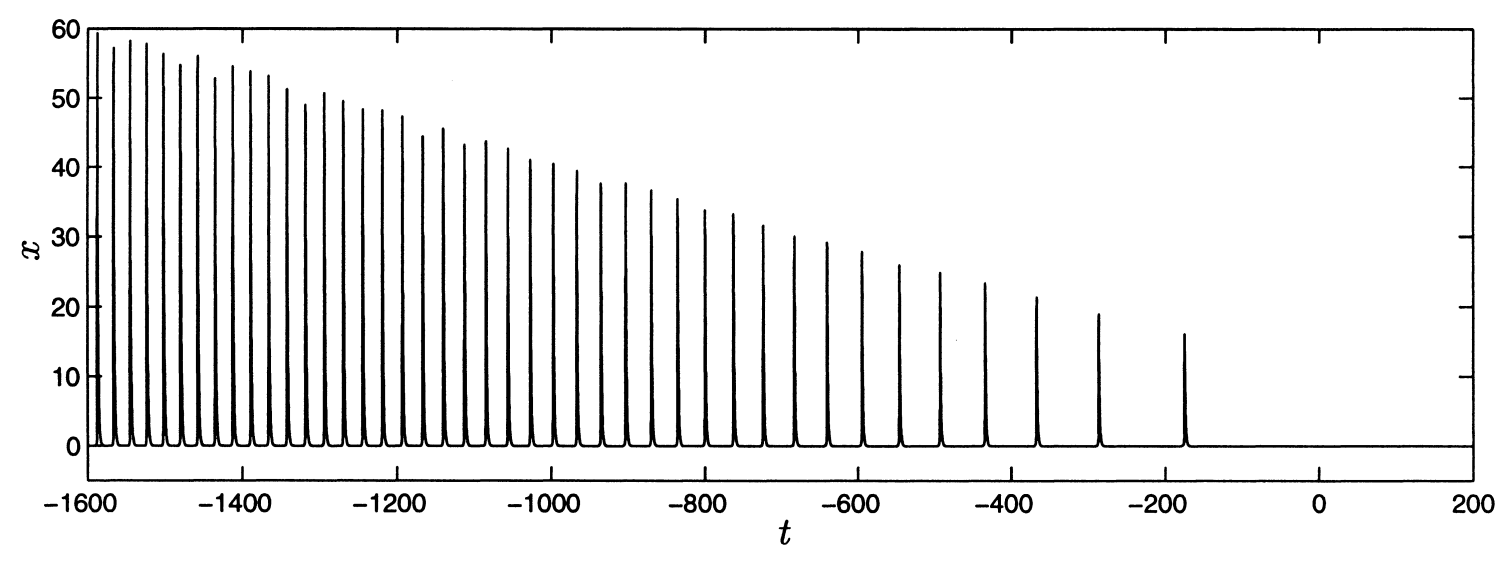

Fig. 4. A typical trajectory of the slowly varying system displaying spike-frequency adaptation and a transition to quiescence. 


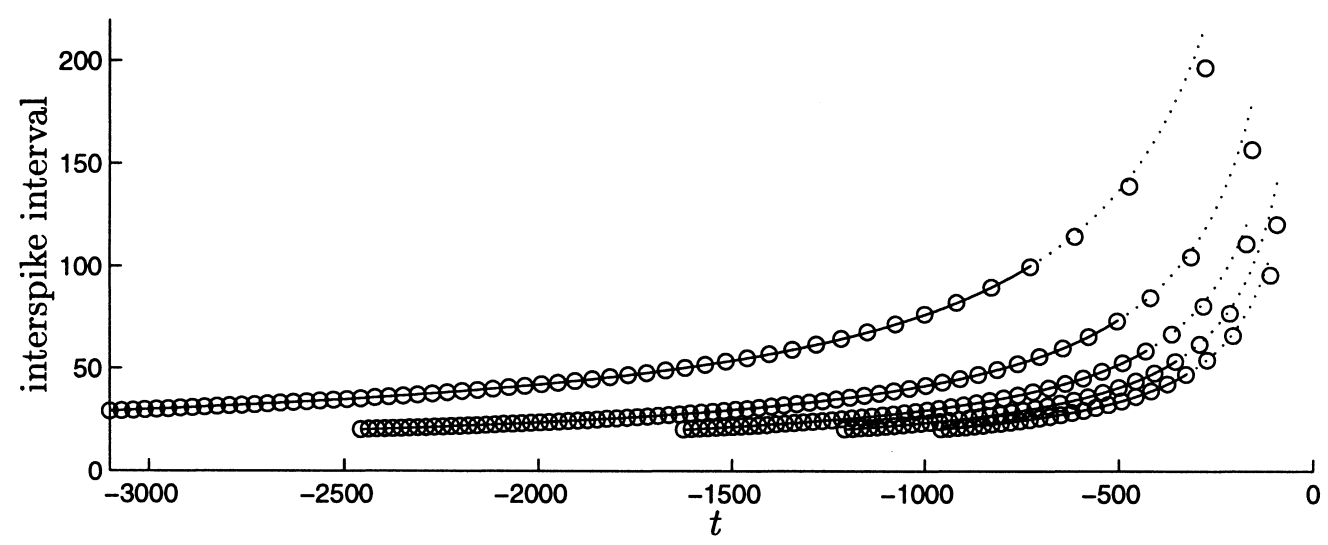

Fig. 5. Interspike intervals for several trajectories slowly approaching the Hopf bifurcation. The six curves, from top to bottom, correspond to trajectories with values of $s$ equal to $2 \times 10^{-4}, 4 \times 10^{-4}, 6 \times 10^{-4}, 8 \times 10^{-4}$ and $10 \times 10^{-4}$, respectively.

The fitted curves are overlaid on the data points in Fig. 5. As can be seen the fits are extremely good, but their extension to the last three spikes (shown as dotted lines) indicates that the last three spikes occur sooner than the curves would predict. As we shall see in Section 6, this is exactly what we would expect.

Even though we observed monotonic increases in the period for this slowly varying system, the complex dynamics near the Hopf-homoclinic transition may create deviations in the interspike intervals from a smooth convex curve. A trajectory may follow a succession of stable periodic orbits of the frozen system, "jumping" from one to the next as saddle-node bifurcations of these periodic orbits are encountered or it may be in a chaotic attractor with returns whose distance from the equilibrium do not vary monotonically. The details of these variations are likely to depend sensitively upon the particular model we study and parameter values within the model. Nonetheless, the general scaling of interspike intervals as a fractional linear function of the time remaining until the Hopf bifurcation is reached, should hold despite this variability.

\section{Subcritical Hopf-homoclinic bifurcation}

Let $X_{\lambda}$ be a smooth vector field depending on the two-dimensional parameter $\lambda$. We list below several hypotheses that we make about the family $X_{\lambda}$. When these hypotheses are satisfied, we say that $X_{\lambda}$ has a generic Hopf-homoclinic bifurcation.

Table 1

Optimal least square parameter values for fits to Eq. (4) for the data in Fig. 5

\begin{tabular}{llll}
\hline$s\left(\times 10^{-4}\right)$ & $a$ & $b$ & $c$ \\
\hline 2 & 4.6836 & 15.6635 & 0.0181 \\
4 & 4.6628 & 15.7961 & 0.0270 \\
6 & 4.7292 & 15.7966 & 0.0330 \\
8 & 4.6765 & 15.9325 & 0.0411 \\
10 & 4.7349 & 15.9139 & 0.0459 \\
Mean & 4.6974 & 15.8205 & 0.0330 \\
Standard deviation & 0.0325 & 0.1085 & 0.0111 \\
\hline
\end{tabular}




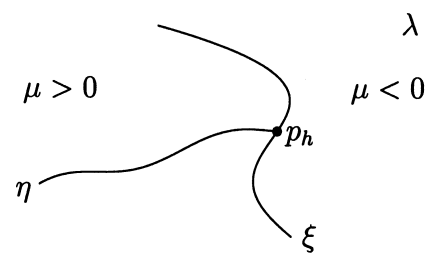

Fig. 6. Bifurcation diagram for the vector field $X_{\lambda} . \xi$ is curve of subcritical Hopf bifurcations giving birth to unstable limit cycles in the region $\mu<0$, and $\eta$ is the curve of homoclinic bifurcations. The Hopf-homoclinic bifurcation occurs at the point $p_{\mathrm{h}}$.

1. There is an equilibrium point $p_{\lambda}$ that varies smoothly with $\lambda$.

(a) There is a simple pair of complex eigenvalues, $\mu(\lambda) \pm \mathrm{i} \omega(\lambda)$, such that $\mu(\lambda)$ passes transversally through zero (i.e., $\nabla_{\lambda} \mu \neq 0$ ) along a curve $\xi$ in the parameter plane and $\omega(\lambda) \neq 0$.

(b) There is a simple eigenvalue $v(\lambda)<0$ such that $v(\lambda)$ is larger (has smaller magnitude) than the real parts of all other eigenvalues except $\mu(\lambda) \pm \mathrm{i} \omega(\lambda)$.

(c) The Hopf bifurcation is subcritical. There is a family of periodic orbits of saddle-type that exist for $\mu(\lambda)<0$. Near the stable equilibrium point $p_{\lambda}$, the stable manifold of these periodic orbits bounds the domain of attraction of $p_{\lambda}$. In the parameter region $\mu \geq 0$, the equilibrium point $p_{\lambda}$ has a two-dimensional unstable manifold on which trajectories diverge from $p_{\lambda}$.

2. In the parameter region $\mu \geq 0$, there is a curve $\eta$ on which the vector field $X_{\lambda}$ has a homoclinic orbit $\sigma_{\lambda}$ for $p_{\lambda}$.

(a) The curve approaches $p_{\lambda}$ in the direction of the eigenvector $v(\lambda)$.

(b) The curve $\eta$ meets the curve $\xi$ transversally at $p_{\mathrm{h}}$. The intersection point is called the Hopf-homoclinic point and we designate the value of $\lambda$ at this point by $h$.

(c) In the product of the parameter and phase spaces, the stable and unstable manifolds of the equilibrium surface $p_{\lambda}$ intersect transversally as the parameter $\lambda$ crosses the curve $\eta$ transversally.

(d) Along the homoclinic curve $\eta, X_{\lambda}$ satisfies an "inclination" property, namely, that there is an invariant subbundle of the tangent bundle of $R^{n}$ along the homoclinic orbit that approaches the three-dimensional subspace spanned by the eigenvectors of $v$ and $\mu(\lambda) \pm \mathrm{i} \omega(\lambda)$.

The bifurcation diagram for $X_{\lambda}$ is shown in Fig. 6 .

There are three phenomena that occur in generic subcritical Hopf-homoclinic bifurcation that we discuss:

1. the existence of chaotic invariant sets;

2. the existence of long, stable periodic orbits;

3. scaling properties for the return of trajectories to the vicinity of $p_{\lambda}$.

Our analysis is based upon geometric study of the return maps for a cross-section to the flow past $p_{\lambda}$. As has become customary, we decompose the return map into a composition of two maps, one representing flow near $p_{\lambda}$ and the second representing a global return. We shall make unsubstantiated approximations in our discussion that simplify the analysis.

Our first approximation is to assume that the analysis of the Hopf-homoclinic bifurcation can be carried out in a three-dimensional submanifold tangent to the eigenspaces of $v$ and $\mu(\lambda) \pm i \omega(\lambda)$ at the equilibrium. In other words, we assume that "strong stable" directions are ignored. Fig. 1 shows a typical trajectory (projected to a three-dimensional manifold) in the parameter region $\mu(\lambda) \geq 0$. The vector field has a normal form in a neighborhood $U$ of the equilibrium point $p_{\lambda}$ that is approximated by the following equations in cylindrical coordinates:

$$
\dot{x}=v x, \quad \dot{r}=\mu r-b x r+a r^{3}, \quad \dot{\theta}=\omega .
$$

By property (1c), $a>0$ so that the Hopf bifurcation is subcritical. Without loss of generality, we also assume $b>0$ and $\omega>0$. We choose two cross-sections $\Sigma_{1}$ and $\Sigma_{2}$ to the homoclinic orbit $\sigma_{\mathrm{h}}$ in $U, \Sigma_{1}$ lying in a plane 
with $x$ constant as $\sigma_{\mathrm{h}}$ approaches $p_{\mathrm{h}}$ and $\Sigma_{2}$ lying in a plane with $\theta$ constant as $\sigma_{\mathrm{h}}$ leaves $p_{\mathrm{h}}$. Note that the vector field is almost tangent to the $\theta$ direction near $p_{\mathrm{h}}$ 's unstable manifold, so the cross-section $\Sigma_{2}$ makes a large angle with the vector field (see Fig. 1). Our second approximation is to assume that the correspondence map that follows trajectories from $\Sigma_{1}$ to their intersection with $\Sigma_{2}$ is replaced by the map associated with the system of equations above.

We remark that the term $-b x r$ above is related to the transverse stability of the stable manifold of $p$. If $b$ is large relative to $|v|$, numerical computations show that large regions of initial data come close to $p$ because they are drawn towards its stable manifold faster than the trajectories approach $p$ along its stable manifold. This behavior cannot persist all the way to $p$ when $p$ is unstable, but the effect is sufficiently dramatic that large regions of parameter space near the Hopf-homoclinic point appear to give approximately homoclinic orbits. Therefore, we have included analysis of this phenomenon in our approximation of the flow past the equilibrium point.

\section{Geometry}

We now discuss the geometric form of the correspondence map $T_{1}$ from $\Sigma_{1}$ to $\Sigma_{2}$; see Fig. 7 . We claim that the image of a circle $r=c_{0}$ in $\Sigma_{1}$ will be a curve in $\Sigma_{2}$ for which the $x$ coordinate decreases monotonically and the
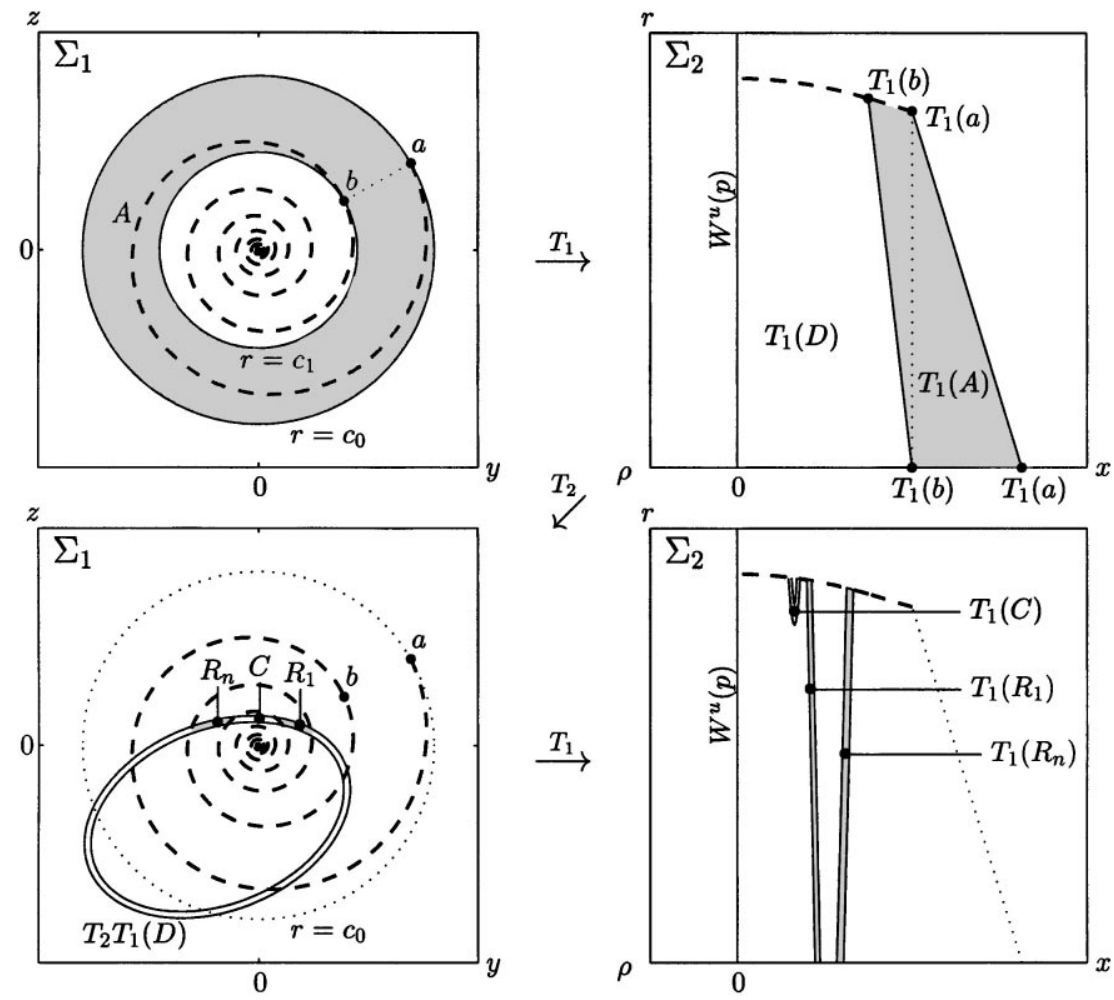

Fig. 7. Geometry of the correspondence maps $T_{1}$ and $T_{2}$. The upper left panel is mapped by $T_{1}$ to the upper right panel, which in turn is mapped to the lower left by $T_{2}$ and finally to the lower right by $T_{1}$ again. The curve $\beta$ in $\Sigma_{1}$ and its image in $\Sigma_{2}$ are drawn as a dashed curve. In the top panels, the region $A$ and its image under $T_{1}$ are shaded, while in the bottom panels, two regions $R_{i}$ and their images under $T_{1}$ are shaded. See text for description. 
$r$ coordinate increases monotonically with the time of passage. This can be seen by noting that the equation for $\theta$ in (5) completely decouples giving $\theta(t)=\theta(0)+\omega t$ and leaving the other two equations independent of $\theta$. All points on the circle $r=c_{0}$ in $\Sigma_{1}$ have identical initial $x$ and $r$ values which means that the circle will remain a circle under the flow until one of the trajectories reaches the lower boundary, $r=\rho$ of $\Sigma_{2}$. After this, the remaining trajectories will intersect with $\Sigma_{2}$ beginning with those that had smaller values of $\theta(0)$ than the first trajectory. The monotonic dependence of this intersection curve is then assured by the fact that $\dot{x}$ is always negative and $\dot{r}$ is positive by the time $\Sigma_{2}$ is reached. Let $a=\left(c_{0}, \theta_{0}\right)$ be the point of discontinuity of $T_{1}$ on the circle $r=c_{0}$, i.e. the initial point of the trajectory which first reaches $\Sigma_{2}$. As we approach the origin along the ray $\theta=\theta_{0}$, the return time increases and we reach additional points of discontinuity for the map $T_{1}$. This is necessarily the case since the ray from $a$ is mapped to a vertical line in $\Sigma_{2}$ which must reach the lower boundary, $r=\rho$, before the ray reaches the origin. Let $b=\left(c_{1}, \theta_{0}\right)$ be the first such point. We assert that the map $T_{1}$ has the geometry depicted in Fig. 7 on the disk $r \leq c_{0}$, denoted $D$. Let $A$ be the annulus $c_{1} \leq r \leq c_{0}$. As $r$ decreases from $c_{0}$ to $c_{1}$, the point of discontinuity for $T_{1}$ along each circle rotates, tracing out a curve $\beta$ that makes one full rotation around $A$ while connecting its two boundary components. Thus $T_{1}(A)$ is discontinuous along $T_{1}(\beta)$, with the limiting values of $T_{1}$ along $\beta$ lying at $\rho$ and the maximum value of $r$ in $T_{1}(A) \subset \Sigma_{2}$. The portion of the disk $D$ not within $A$ is mapped to a region between $T_{1}(A)$ and the line $\Sigma_{2} \cap W^{u}(p)$. Note that since the minimum passage time, $t_{\mathrm{m}}$, of $T_{1}$ is large, $T_{1}(D)$ is very thin in the $x$ direction, with distance from the unstable manifold of $p$ bounded by $\left|x(0) \exp \left(v t_{\mathrm{m}}\right)\right|$.

Next consider the correspondence map $T_{2}$ from $\Sigma_{2}$ to $\Sigma_{1}$. This map is regular and $\gamma=T_{2}\left(\Sigma_{2} \cap W^{u}(p)\right)$ is a smooth closed curve in $\Sigma_{1}$. For parameter values with a homoclinic orbit, $\gamma$ passes through the origin. $T_{2}$ maps $T_{1}(D)$ onto a closed ring in $\Sigma_{1}$ with the curve $\gamma$ as one boundary. Since the width of $T_{1}(D)$ is so small, we can approximate $T_{2} T_{1}(D)$ by its projection onto $\gamma$.

We finally describe some of the chaotic dynamics and stable periodic orbits associated with the Hopf-homoclinic bifurcation. The analysis is identical to that used to describe the formation of homoclinic tangencies for discrete diffeomorphisms (Guckenheimer and Holmes [6]). Extend $\beta$ to be $T_{1}^{-1}(\rho) \cap D$. The extended curve $\beta$ is a spiral emanating from $W^{\mathrm{s}}(p) \cap \Sigma_{1}$. If $\gamma$ comes close enough to $W^{\mathrm{s}}(p) \cap \Sigma_{1}, T_{2} T_{1}(D)$ is a thin strip that intersects $\beta$ many times, partitioning $T_{2} T_{1}(D)$ into many subsets. There is one central subset $C$ with the property that $C$ together with the segment cut from $\beta$ does not enclose the origin. Each remaining subset $R_{i}$ cuts out a segment of $\beta$ so that the union of the two does enclose the origin. $T_{1}$ then maps each segment $R_{i}$ to a narrow strip stretching across $T_{1}(D)$ in $\Sigma_{2}$ and the composite map $T_{2} T_{1}$ takes each $R_{i}$ into a strip that spirals once around $T_{2} T_{1}(D)$. The stretching increases lengths along the direction of $\gamma$. On $R_{i} \cup R_{i+1}, T_{2} T_{1}$ forms a horseshoe with chaotic trajectories [6]. $T_{1}$ maps the central segment, $C$, to a curved strip returning to $T_{1}(\beta)$ in $\Sigma_{2}$, while the composite map $T_{2} T_{1}$ has a critical point on $C$, so that $T_{2} T_{1}(C)$ is folded along $T_{2} T_{1}(D)$.

While the presence of a horseshoe identifies chaotic trajectories of $X_{\lambda}$, it does not tell us what sort of attractors we are likely to encounter. We note that the flow past the equilibrium $p$ is volume contracting since the divergence, $v+2 \mu-2 b x+4 a r^{3}$, of the approximating vector field is negative when $r$ is small (we are assuming $\mu$ is small, i.e. close to the Hopf bifurcation). This implies that the return map $T_{2} T_{1}$ of $\Sigma_{1}$ will be area contracting. This leads us to expect that there will be chaotic attractors for some parameter values and stable periodic orbits for others. If a parameter is varied so that the distance from $\gamma$ to the origin decreases, then the fold of $T_{2} T_{1}(C)$ sweeps around $T_{2} T_{1}(D)$ (i.e. $T_{1}(C)$ stretches down across $T_{1}(D)$, see Fig. 7) until the central segment becomes tangent to $\beta$, creating a new pair of subsets $R_{i}$ and a new central segment. This process creates new periodic orbits, some of which are stable at their creation as described in Section 6.6 of Guckenheimer and Holmes [6]. The return map $T_{2} T_{1}$ has homoclinic tangencies and the theory of Newhouse [8] implies that there are parameter values for which $X_{\lambda}$ has wild hyperbolic sets and infinitely many stable periodic orbits. 


\section{Travel time past the equilibrium point}

In this section we develop asymptotic expressions for the travel times of trajectories returning to the vicinity of $p_{\lambda}$, as $\lambda$ varies slowly across the Hopf bifurcation curve, $\xi$, near the Hopf-homoclinic point, $h$.

Instead of using the cross-section, $\Sigma_{2}$, on which $\theta$ is a constant, here we shall redefine $\Sigma_{2}$ to be a cylindrical shell at a fixed radius, $r_{2}$. This allows us to ignore the $\theta$ component completely and restrict our analysis to the half-plane consisting of the stable direction, $x$ and the radial direction, $r$, tangent to the center manifold of the equilibrium point. We are primarily interested in how the travel time will vary over successive passes through the neighborhood of $p_{\lambda}$ as the Hopf bifurcation curve is slowly approached, however, we are also interested in obtaining an expression for the travel time when the real part of the Hopf eigenvalue, $\mu$, is fixed. Since the mathematics is initially the same for both cases, we begin by writing $\mu=\mu_{0}-s t$. Then, by setting $s$ to zero, $\mu$ becomes a constant and when $\mu_{0}$ is set to zero with $s>0, \mu$ will decrease linearly with time until the Hopf bifurcation curve, $\xi$, is crossed at time $t=0$, after which the equilibrium point will give birth to unstable limit cycles in the $x=0$ plane. Thus the model in the $(x, r)$ plane becomes

$$
\frac{\mathrm{d} x}{\mathrm{~d} t}=v x, \quad \frac{\mathrm{d} r}{\mathrm{~d} t}=\left(\mu_{0}-s t\right) r-b x r+a r^{3}, \quad t \leq 0,
$$

where the constants $a, b$ and $s$ are positive, $v$ is negative and $\mu=\mu_{0}-s t$ is non-negative. Scaling the variables and constants as

$$
\hat{x}=\frac{b}{-v} x, \quad \hat{r}=\sqrt{\frac{a}{-v}} r, \quad \hat{t}=-v t, \quad \hat{s}=\frac{s}{v^{2}}, \quad \hat{\mu}_{0}=\frac{\mu_{0}}{-v}
$$

yields the dimensionless system (from which we drop the hats for notational convenience):

$$
\frac{\mathrm{d} x}{\mathrm{~d} t}=-x, \quad \frac{\mathrm{d} r}{\mathrm{~d} t}=\left(\mu_{0}-s t\right) r-x r+r^{3}, \quad t \leq 0
$$

We consider trajectories of system (7) starting at time $t_{1}$ at the cross-section $\Sigma_{1}$ (defined by $x=x_{1}$ ) and ending at time $t_{2} \leq 0$ at the cross-section $\Sigma_{2}$ (defined by $r=r_{2}$ ); see Fig. 8. If $t_{2}=0$, then the trajectory represents in some sense the last possible passage through the region of interest since, for positive $t$, the equilibrium point becomes stable and attractive. For a single trajectory, we label the points at the intersections with the cross-sections as

$$
\begin{aligned}
& \Sigma_{1}: \quad x\left(t_{1}\right)=x_{1}, \quad r\left(t_{1}\right)=r_{1}, \\
& \Sigma_{2}: \quad x\left(t_{2}\right)=x_{2}, \quad r\left(t_{2}\right)=r_{2},
\end{aligned}
$$

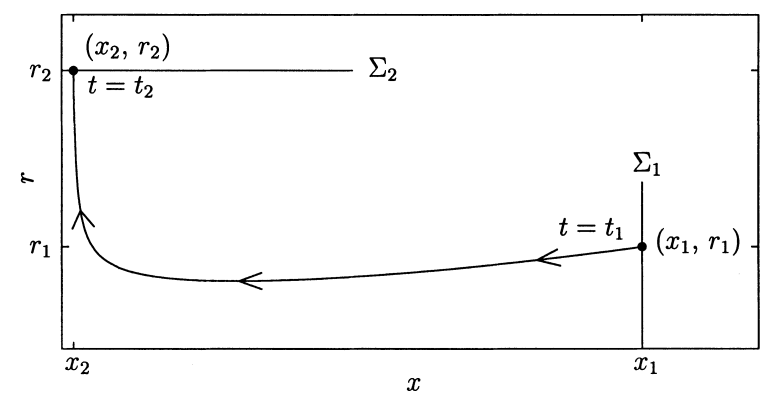

Fig. 8. Phase plane for system (7). 
where $x_{1}>x_{2}>0$ and $r_{2}>r_{1}>0$. The first differential equation in (7) is independent of $r$ and thus decoupled from the second. Solving this first equation with either initial conditions (8) or (9) gives

$$
x(t)=x_{1} \mathrm{e}^{-\left(t-t_{1}\right)} \quad \text { or } \quad x(t)=x_{2} \mathrm{e}^{-\left(t-t_{2}\right)},
$$

respectively. Since by definition the same trajectory satisfies both (8) and (9), we may invert the two equations in (10) to obtain the following expressions for the time $t$ :

$$
t=t_{1}-\ln \left(\frac{x}{x_{1}}\right)=t_{2}-\ln \left(\frac{x}{x_{2}}\right) .
$$

This inversion allows us to view $x$ as the independent variable. By the chain rule we have

$$
\frac{\mathrm{d} r}{\mathrm{~d} t}=\frac{\mathrm{d} x}{\mathrm{~d} t} \frac{\mathrm{d} r}{\mathrm{~d} x}=-x \frac{\mathrm{d} r}{\mathrm{~d} x},
$$

so that our system of equations reduces to

$$
\begin{array}{ll}
\frac{\mathrm{d} r}{\mathrm{~d} x}=\left(\frac{s t-\mu_{0}}{x}+1-\frac{r^{2}}{x}\right) r, & \\
t=t_{2}-\ln \left(\frac{x}{x_{2}}\right), & x_{2} \leq x \leq x_{1}, \\
r\left(x_{1}\right)=r_{1}, &
\end{array}
$$

where the constant $x_{2}$ is defined by $r\left(x_{2}\right)=r_{2}$.

We are concerned with obtaining estimates for the traversal time, $P$, from the cross-section $\Sigma_{1}$ to the cross-section $\Sigma_{2}$, that is, estimates for $P=t_{2}-t_{1}$. From (11), this time is given by

$$
P=t_{2}-t_{1}=\ln \left(\frac{x_{1}}{x_{2}}\right) .
$$

In particular, we shall be interested in determining the behavior of $P$ as $-t_{2}$ decreases while $r_{1}$ is close to zero, that is, as the distance to the Hopf bifurcation decreases while the initial point of the trajectory remains close to the stable manifold, $r=0$, of the equilibrium point. Our analysis shall take the following course. First we shall obtain some inequalities for $P$ in terms of an integral involving the unknown trajectory $r(x)$. We shall then use these inequalities to obtain upper and lower bounds for $P$ in the cases $s=0$ and $s \neq 0$ and finally we shall investigate the functional form of $P$ when the initial time is sufficiently distant from the bifurcation to allow enough time for the trajectory to make multiple passes through the region near the equilibrium point.

\subsection{An integral inequality for the travel time}

Formally integrating system (12) gives

$$
\begin{aligned}
\int_{r_{1}}^{r_{2}} \frac{\mathrm{d} r}{r} & =\int_{x_{1}}^{x_{2}}\left(\frac{s t_{2}-\mu_{0}-s \ln \left(x / x_{2}\right)}{x}+1-\frac{r^{2}}{x}\right) \mathrm{d} x, \\
\ln \left(\frac{r_{2}}{r_{1}}\right) & =\left(s t_{2}-\mu_{0}\right) \ln \left(\frac{x_{2}}{x_{1}}\right)+\frac{s}{2}\left[\ln \left(\frac{x_{1}}{x_{2}}\right)\right]^{2}+x_{1}\left(\frac{x_{2}}{x_{1}}-1\right)-\int_{x_{1}}^{x_{2}} \frac{r^{2}}{x} \mathrm{~d} x \\
& =\left(\mu_{0}-s t_{2}\right) P+\frac{s}{2} P^{2}-x_{1}\left(1-\mathrm{e}^{-P}\right)-\int_{x_{1}}^{x_{2}} \frac{r^{2}}{x} \mathrm{~d} x .
\end{aligned}
$$


Defining $K$ as

$$
K=\int_{x_{2}}^{x_{1}} \frac{r^{2}}{x} \mathrm{~d} x
$$

Eq. (13) may be written as

$$
K=\ln \left(\frac{r_{2}}{r_{1}}\right)+x_{1}\left(1-\mathrm{e}^{-P}\right)+\left(s t_{2}-\mu_{0}\right) P-\frac{s}{2} P^{2} .
$$

We now turn our attention to estimates for $K$. Since $r<r_{2}$ on $\left(x_{2}, x_{1}\right)$, it is clear that

$$
K<\int_{x_{2}}^{x_{1}} \frac{r_{2}^{2}}{x} \mathrm{~d} x=r_{2}^{2} \ln \left(\frac{x_{1}}{x_{2}}\right)=r_{2}^{2} P .
$$

Further, since $\mathrm{d} r / \mathrm{d} x<r$, it follows that if $r\left(x_{1}\right)=r_{1}$ then $r(x)>r_{1} \mathrm{e}^{\left(x-x_{1}\right)}$ for $x<x_{1}$. Consequently, the minimum value obtained by $r$ on $\left[x_{2}, x_{1}\right]$ satisfies

$$
r_{\min }>r_{1} \mathrm{e}^{\left(x_{2}-x_{1}\right)}=r_{1} \exp \left(-x_{1}\left(1-\mathrm{e}^{-P}\right)\right)>r_{1} \mathrm{e}^{-x_{1}},
$$

where we have used the fact that $x_{2} / x_{1}=\mathrm{e}^{-P}<1$. Therefore, a lower bound for $K$ is given by

$$
K>\int_{x_{2}}^{x_{1}} \frac{r_{\min }^{2}}{x} \mathrm{~d} x>\int_{x_{2}}^{x_{1}} \frac{r_{1}^{2} \mathrm{e}^{-2 x_{1}}}{x} \mathrm{~d} x=r_{1}^{2} \mathrm{e}^{-2 x_{1}} P .
$$

Substituting inequalities (16) and (17) into (15) gives the relationship

$$
0<r_{1}^{2} \mathrm{e}^{-2 x_{1}} P<K=\ln \left(\frac{r_{2}}{r_{1}}\right)+x_{1}\left(1-\mathrm{e}^{-P}\right)+\left(s t_{2}-\mu_{0}\right) P-\frac{s}{2} P^{2}<r_{2}^{2} P .
$$

\subsection{Estimates for the frozen system}

In the case that $s=0$, the system is said to be frozen. If $\mu_{0}$ is zero, then the system is frozen at the Hopf bifurcation, whereas if $\mu_{0}$ is positive, the system is frozen at some fixed distance from the Hopf bifurcation curve. With $s$ set to zero, system (12) becomes

$$
\frac{\mathrm{d} r}{\mathrm{~d} x}=\left(\frac{-\mu_{0}}{x}+1-\frac{r^{2}}{x}\right) r, \quad r\left(x_{1}\right)=r_{1}, \quad x_{2} \leq x \leq x_{1},
$$

where $x_{2}$ is defined by $r\left(x_{2}\right)=r_{2}$. Consider the system

$$
\frac{\mathrm{d} \hat{r}}{\mathrm{~d} x}=-\frac{\mu_{0} \hat{r}+\hat{r}^{3}}{x}, \quad \hat{r}\left(x_{2}\right)=r_{2},
$$

where, compared with (19), the term $r$, which is positive, has been dropped from the differential equation and the starting point has been set as the intersection of the trajectory with the cross-section $\Sigma_{2}$ rather than with $\Sigma_{1}$. Since $\mathrm{d} \hat{r} / \mathrm{d} x<\mathrm{d} r / \mathrm{d} x$, it follows immediately that $\hat{r}(x)<r(x)$ on $\left(x_{2}, x_{1}\right)$; see Fig. 9. Assuming for now that $\mu_{0} \neq 0$, we may solve (20) to obtain

$$
\hat{r}^{2}(x)=\frac{\mu_{0}}{\left(1+\left(\mu_{0} / r_{2}^{2}\right)\right)\left(x / x_{2}\right)^{2 \mu_{0}}-1} .
$$




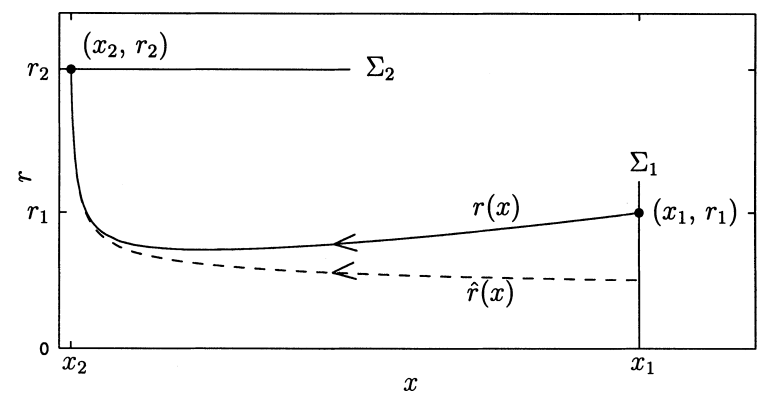

Fig. 9. Trajectories of system (19) (solid line) and (20) (dashed line).

Using the facts that $\ln \left(x_{1} / x_{2}\right)=P$ and $\hat{r}^{2}\left(x_{1}\right)<r_{1}^{2}$, we get

$$
\hat{r}^{2}\left(x_{1}\right)=\frac{\mu_{0}}{\left(1+\left(\mu_{0} / r_{2}^{2}\right)\right) \mathrm{e}^{2 \mu_{0} P}-1}<r_{1}^{2},
$$

which implies

$$
P>\frac{1}{2 \mu_{0}} \ln \left(\frac{1+\left(\mu_{0} / r_{1}^{2}\right)}{1+\left(\mu_{0} / r_{2}^{2}\right)}\right) .
$$

Further, since $\hat{r}(x)<r(x)$ we may substitute $\hat{r}$ for $r$ into (14) and integrate to obtain

$$
K>\frac{1}{2} \ln \left[\left(1+\frac{\mu_{0}}{r_{2}^{2}}\right)\left(\frac{x}{x_{2}}\right)^{2 \mu_{0}}-1\right]-\mu_{0} \ln \left(\frac{x}{x_{2}}\right)||_{x_{2}}^{x_{1}}>\frac{1}{2} \ln \left[\left(1+\frac{\mu_{0}}{r_{2}^{2}}\right) \mathrm{e}^{2 \mu_{0} P}-1\right]-\mu_{0} P-\frac{1}{2} \ln \left(\frac{\mu_{0}}{r_{2}^{2}}\right),
$$

where we have again used $\ln \left(x_{1} / x_{2}\right)=P$. Since $1-\mathrm{e}^{-P}<1$, from (15) we have

$$
K<\ln \left(\frac{r_{2}}{r_{1}}\right)+x_{1}-\mu_{0} P
$$

which we now combine with the above inequality and re-arrange to give

$$
P<\frac{1}{2 \mu_{0}} \ln \left(\frac{1+\left(\mu_{0} / r_{1}^{2} \mathrm{e}^{-2 x_{1}}\right)}{1+\left(\mu_{0} / r_{2}^{2}\right)}\right) .
$$

In the limit as $\mu_{0} \rightarrow 0$, the bounds (22) and (23) approach

$$
\begin{aligned}
& P>\frac{1}{2}\left(\frac{1}{r_{1}^{2}}-\frac{1}{r_{2}^{2}}\right), \\
& P<\frac{1}{2}\left(\frac{\mathrm{e}^{2 x_{1}}}{r_{1}^{2}}-\frac{1}{r_{2}^{2}}\right),
\end{aligned}
$$

respectively. (These last two bounds can also be obtained from system (20) in the same manner as (22) and (23) by assuming $\mu_{0}=0$ initially.) The bounds for $\mu_{0} \neq 0$ show that when the real part of the Hopf eigenvalue is fixed away from zero, the travel time between the two cross-sections is proportional to $1 / \mu_{0}$ modified by a logarithmic factor involving $\mu_{0}$. The logarithmic factor's influence is determined by the relative sizes of $r_{1}$ and $r_{2}$. Considering the lower bound (analysis of the upper bound is similar), this factor increases to $\ln \left(r_{2} / r_{1}\right)$, in the limit as $\mu_{0}$ becomes 


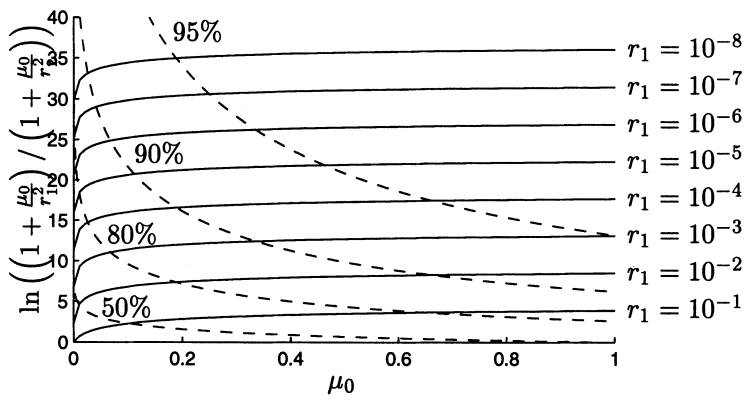

Fig. 10. Dependence of the logarithmic factor on the Hopf eigenvalue and the distance to the homoclinic trajectory. The dashed curves indicate the places at which the logarithmic factor reaches the indicated percentage of its limiting value.

large. If $r_{1}$ is close to $r_{2}$, then the logarithmic factor is small but varies considerably in relative size with $\mu_{0}$. If $r_{1}$ is much smaller than $r_{2}$, then the factor is larger but displays less relative variance with $\mu_{0}$. This analysis is shown in Fig. 10, where we have plotted the value of the logarithmic factor as a function of $\mu_{0}$ for various values of $r_{1}\left(r_{2}\right.$ fixed at 1). Overlaid on the plot are several percentile curves which show the location where the logarithmic factor reaches $50,80,90$ and $95 \%$ of its limiting value. Notice that when $r_{1}$ is small, the logarithmic factor is relatively more constant for smaller values of $\mu_{0}$. As a consequence, the travel time dependence for near homoclinic trajectories ( $r_{1}$ small) will be nearly proportional to $1 / \mu_{0}$ and will only deviate substantially if $\mu_{0}$ is very small.

\subsection{Estimates for the varying system}

Here, we are concerned with analyzing system (12) in the case $\mu_{0}=0$ and $s>0$ so that the real part of the Hopf eigenvalue crosses from positive to negative at time zero. In the previous section, where the system was frozen, we obtained bounds for $P$ by estimating the unknown trajectory $r(x)$ with a known trajectory, $\hat{r}(x)$, which was the solution to a differential equation similar to that satisfied by $r(x)$. In the varying case, it is more difficult to come up with a similar differential equation that is solvable and which allows us to isolate $P$ from the other parameters. Instead, in this section, we replace $r(x)$ with a constant function to obtain our results.

The inequalities of (18) were obtained by replacing $r(x)$ in $K$ with either a lower bound, $r_{1} \mathrm{e}^{-x_{1}}$, or an upper bound, $r_{2}$. Using the fact that $0<\left(1-\mathrm{e}^{-P}\right)<1$ and setting $\mu_{0}$ to 0 , the two inequalities of (18) become

$$
\begin{aligned}
& -\frac{s}{2} P^{2}+\left(s t_{2}-r_{2}^{2}\right) P+\ln \left(\frac{r_{2}}{r_{1}}\right)<0, \\
& 0<-\frac{s}{2} P^{2}+\left(s t_{2}-r_{1}^{2} \mathrm{e}^{-2 x_{1}}\right) P+\ln \left(\frac{r_{2}}{r_{1}}\right)+x_{1} .
\end{aligned}
$$

Both of the above quadratic functions of $P$ have exactly one positive real root and represent parabolas opening downward. We are only interested in positive values of $P$, thus inequality (26) gives

$$
P>\frac{1}{s}\left(\left(s t_{2}-r_{2}^{2}\right)+\sqrt{\left(s t_{2}-r_{2}^{2}\right)^{2}+2 s \ln \left(\frac{r_{2}}{r_{1}}\right)}\right),
$$

while (27) gives

$$
P<\frac{1}{s}\left(\left(s t_{2}-r_{1}^{2} \mathrm{e}^{-2 x_{1}}\right)+\sqrt{\left(s t_{2}-r_{1}^{2} \mathrm{e}^{-2 x_{1}}\right)^{2}+2 s\left(\ln \left(\frac{r_{2}}{r_{1}}\right)+x_{1}\right)}\right) .
$$


In the limit as $s$ approaches zero, these bounds give

$$
\begin{aligned}
& P>\frac{1}{r_{2}^{2}} \ln \left(\frac{r_{2}}{r_{1}}\right)\left[1-\frac{1}{2 r_{2}^{4}}\left(\ln \left(\frac{r_{2}}{r_{1}}\right)-2 t_{2} r_{2}^{2}\right) s+\mathrm{O}\left(s^{2}\right)\right], \\
& P<\frac{\mathrm{e}^{2 x_{1}}}{r_{1}^{2}} \ln \left(\frac{r_{2}}{r_{1}}\right)\left[1-\frac{\mathrm{e}^{4 x_{1}}}{2 r_{1}^{4}}\left(\ln \left(\frac{r_{2}}{r_{1}}\right)-2 t_{2} \frac{r_{1}^{2}}{\mathrm{e}^{2 x_{1}}}\right) s+\mathrm{O}\left(s^{2}\right)\right],
\end{aligned}
$$

which converge as $r_{1} \rightarrow 0$ in the regions $s<1 / \ln \left(r_{2} / r_{1}\right)$ and $s<r_{1}^{4} / \ln \left(r_{2} / r_{1}\right)$, respectively. We see then that as $s$ approaches zero, these bounds on $P$ do not approach the bounds obtained for the frozen system. Inequalities (30) and (31), whose leading terms are of the order $\ln \left(r_{2} / r_{1}\right)$ and $r_{1}^{-2} \ln \left(r_{2} / r_{1}\right)$, provide only a wide bracket around the frozen system bounds whose leading terms are both $r_{1}^{-2}$. In Appendix A we address this issue by replacing $r(x)$ with the solution of a similar differential equation which, although it does not allow us to isolate $P$ explicitly, does at least allow us to obtain Taylor series expressions for the bounds on $P$ which uniformly approach the frozen system bounds as $s$ approaches zero, however, our main goal is to obtain a simple closed form estimate for the behavior of $P$ and its dependence on the time remaining until the Hopf bifurcation, $-t_{2}$. By the mean value theorem for integrals, there is a constant, $R$ (depending on $r_{1}, r_{2}, x_{1}, s$ and $t_{2}$ ) such that the integral $K$ is equal to $R^{2} \ln \left(x_{1} / x_{2}\right)$. Clearly, $r_{1} \mathrm{e}^{-x_{1}}<R<r_{2}$. Although in general $R$ depends on $t_{2}$, it can be shown that the variation of $R^{2}$ with $t_{2}$ is bounded below by the linear function

$$
R^{2}>R_{0}^{2}+\left(1-\frac{R_{0}^{2}}{r_{2}^{2}}\right) s t_{2}
$$

where $R_{0}$ is the value of $R$ when $t_{2}=0$. Further, numerical calculations reveal that the variation of $R^{2}$ with $t_{2}$ decreases as $s$ becomes small. In our numerical example from Section 2, when $s$ was less than $10^{-4}, R^{2}$ showed almost no variation with $t_{2}$; when $s=10^{-4}, R^{2}$ varied nearly linearly with $t_{2}$ with a slope of about $2 \times 10^{-5}$; and for larger values of $s$, the variation of $R^{2}$ with $t_{2}$ was nonlinear but bounded below by the estimate given above. In any event, using similar analysis as above, we get

$$
P \approx \frac{1}{s}\left(\left(s t_{2}-R^{2}\right)+\sqrt{\left(s t_{2}-R^{2}\right)^{2}+2 s\left(\ln \left(\frac{r_{2}}{r_{1}}\right)+\theta\right)}\right),
$$

where $0 \leq \theta \leq x_{1}$. We shall use this estimate to obtain a simple closed form for $P\left(t_{2}\right)$ in the next section.

\section{Variation of the travel time over multiple passes}

In the framework of this section, the Hopf bifurcation curve is crossed at time $t=0$ and $P$ represents the time taken for the trajectory to pass through the nearby region, $U$, of the equilibrium point, leaving this region by crossing $\Sigma_{2}$ at time $t_{2} \leq 0$. We now assume that after leaving the region $U$, the trajectory quickly travels around and re-enters $U$ by crossing $\Sigma_{1}$ again (see Fig. 1). It is easy to see from Fig. 8 that as $r_{1}$ decreases to zero, trajectories entering at $\left(x_{1}, r_{1}\right)$ will cross $\Sigma_{2}$ with smaller values of $x_{2}$. The same effect ( $x_{2}$ decreases) is also caused by increasing $t_{2}$ to zero. This latter fact is seen by analyzing system (12) where, if $t_{2}$ increases to zero, the term $s t r / x$, which is negative, will become smaller in magnitude at $\Sigma_{2}$, indicating that the slope, $\mathrm{d} r / \mathrm{d} x$, of a trajectory passing through $\left(x_{2}, r_{2}\right)$ will be more positive, and thus, for it to have started at the same point $\left(x_{1}, r_{1}\right), x_{2}$ must necessarily be smaller. Since $P=\ln \left(x_{1} / x_{2}\right)$, as $x_{2}$ decreases ( $r_{1}$ decreases or $t_{2}$ increases) the trajectory will spend more time within the region $U$. Since these systems show a substantial contraction toward the stable manifold, the value of 
$r_{1}$ will be very small even when the system is still a considerable distance from the Hopf bifurcation curve. Thus, even if $r_{1}$ increases from one pass to the next, this increase is small and the subsequent decrease in $P$ is more than balanced by the increase in $P$ caused by $t_{2}$ increasing. Assuming that $P$ increases with each pass through $U$, it follows that if $P>-t_{2}$ for any given pass, then that pass will be the last complete passage through $U$ before the Hopf bifurcation occurs. In analysis of data from the LP cell model (and also of experimental data from the real LP cell), a single oscillation is identified by watching when the cell crosses a particular voltage threshold. This corresponds to a trajectory of our model system crossing the section $\Sigma_{2}$. The period of each oscillation is set as the time from the previous crossing to the current one. Consequently, at most one oscillation will be detected which satisfies $P>-t_{2}$ and this oscillation will be the final one before the transition to quiescence occurs.

Since we wish to determine how $P$ changes over several successive passes through $U$, we are interested in the regime

$$
P \approx \frac{-t_{2}}{M},
$$

where $M$ roughly corresponds to the maximum number of remaining oscillations. For example, if $M=3$ then once $P$ gets as large as $-t_{2} / 3$, we would expect to see only two or three more oscillations. Combining (34) with the approximation of $P(33)$, we get the approximate inequality

$$
\left(s t_{2}-R^{2}\right)+\sqrt{\left(s t_{2}-R^{2}\right)^{2}+2 s\left(\ln \left(\frac{r_{2}}{r_{1}}\right)+\theta\right)} \approx \frac{-s t_{2}}{M},
$$

which becomes

$$
\sqrt{\left(s t_{2}-R^{2}\right)^{2}+2 s\left(\ln \left(\frac{r_{2}}{r_{1}}\right)+\theta\right)} \approx-\left(1+\frac{1}{M}\right) s t_{2}+R^{2} \approx\left(1+\frac{1}{M}\right)\left(-s t_{2}+R^{2}\right)
$$

implying

$$
2 s\left(\ln \left(\frac{r_{2}}{r_{1}}\right)+\theta\right) \approx\left(\frac{2 M+1}{M^{2}}\right)\left(-s t_{2}+R^{2}\right)^{2} .
$$

The estimate (35) gives a relationship between the magnitudes of the two terms under the radical sign in the approximation for $P$ given by (33). If we define $A=R^{2}-s t_{2}$ (note: $\left.A>0\right)$ and $B=2 s\left(\ln \left(r_{2} / r_{1}\right)+\theta\right)$, then the estimate (35) says that $B \approx\left(2 M+1 / M^{2}\right) A^{2}$ and (33) may be written as

$$
P \approx \frac{1}{S}\left(-A+\sqrt{A^{2}+B}\right) .
$$

We now use a Taylor series to give

$$
\begin{aligned}
-A+\sqrt{A^{2}+B} & =\sum_{n=1}^{\infty} \frac{(-1)^{n}[(2 n-3)(2 n-5) \cdots(-1)] B^{n}}{2^{n} n ! A^{2 n-1}} \\
& \approx \frac{B}{2 A}\left(\sum_{n=1}^{\infty} \frac{(-1)^{n}[(2 n-3)(2 n-5) \cdots(-1)](2 M+1)^{n}}{2^{n-1} n ! M^{2 n}}\right)
\end{aligned}
$$

This series is convergent provided $M \geq 1+\sqrt{2}$; Fig. 11 shows the convergent value for the sum as a function of $M$. At $M=1+\sqrt{2}$, the sum is approximately 0.8284 and as $M$ becomes large, the sum approaches 1 . It follows 


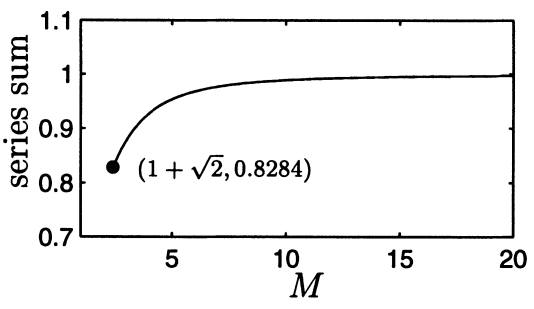

Fig. 11. Convergent values of the sum in (37).

then that provided there are three or more oscillations before the transition to quiescence, the traversal time through the region near the Hopf-homoclinic bifurcation is approximately given by

$$
P \approx C \frac{\ln \left(r_{2} / r_{1}\right)+\theta}{R^{2}-s t_{2}},
$$

where $C$ is a number between 0.8284 and 1 . We argued in the previous section that $R^{2}$ is nearly independent of $t_{2}$ when $s$ is very small and is roughly linearly dependent as $s$ becomes a bit larger. Even if the dependence is approximately linear, it can be absorbed into the $s t_{2}$ term in (38) by a redefinition of $s$. The bound (32) shows that this linear dependence has slope less than $s$ so that the effect on (38) is a value of $s$ perhaps smaller than the true speed of approach but in all cases still positive. Thus $P$ has a fractional linear dependence on $t_{2}$ with $P$ increasing as $t_{2}$ increases toward zero.

In a slowly varying system which passes close to a Hopf-homoclinic bifurcation, the homoclinic curve, $\eta$, could in general be crossed zero or more times before the Hopf bifurcation curve $\xi$ is crossed (see Fig. 6). Nonetheless, with probability 1 , any trajectory starting from a random initial point before the Hopf bifurcation will, at each intersection with $\Sigma_{1}$ have a positive value of $r_{1}$. That is, the trajectory will not coincide with the stable manifold of the equilibrium point. Generically we expect that at each intersection of a specific trajectory with $\Sigma_{1}$, the value of $r_{1}$ will not be significantly altered and thus the terms involving $r_{1}$ in (38) can be replaced by constants. Consequently, with each pass through $U$, the magnitude of $t_{2}$ will be decreasing and the traversal time, $P$, will behave like the fractional linear function $1 /(b-c t)$ over a relatively large time range before the final few oscillations prior to the transition to quiescence. If we now add a bounded constant, $a$, representing the time taken for the trajectory to loop around outside of $U$ from $\Sigma_{2}$ back to $\Sigma_{1}$, we obtain the expression (2) which we used in [5] for fitting to interspike interval data from trajectories influenced by this bifurcation.

\section{Concluding remark}

This paper was motivated by the seeming appearance of near homoclinic orbits arising as a consequence of subcritical Hopf bifurcation. Our analysis explains why this occurs: large regions of the phase space of a system undergoing subcritical Hopf bifurcation flow close to the stable manifold of the bifurcating equilibrium point. We believe that this phenomenon is quite general and present in many other applications besides the neural models studied in this paper (see, for example [4]). The attractor that appears subsequent to subcritical Hopf bifurcation is often a long periodic orbit, though if one is in the immediate vicinity of the Hopf-homoclinic codimension 2 bifurcation, chaotic attractors of Silnikov type are possible. Our results give estimates for the size of these regions. Outside the parameter regions close to homoclinic bifurcation, we demonstrate that the period of the periodic orbits is approximated by a fractional linear function of the distance from the bifurcation. This asymptotic estimate gives a criterion that can be used to identify the occurrence of subcritical Hopf bifurcation in time series data. 


\section{Appendix A}

To obtain estimates on the traversal time which uniformly approach the frozen system estimates as $s \rightarrow 0$, we consider the system

$$
\frac{\mathrm{d} \tilde{r}}{\mathrm{~d} x}=-\left(\frac{s\left(P-t_{2}\right)}{x}+\frac{\tilde{r}^{2}}{x}\right) \tilde{r}, \quad \tilde{r}\left(x_{2}\right)=r_{2} .
$$

Notice that as $s \rightarrow 0$, this system collapses to the $\hat{r}$ system (20), analyzed in Section 5.2. Comparing system (A.1) to system (12), since $P-t_{2} \leq-t \leq 0$, it follows that $\tilde{r}(x)<r(x)$ on $\left(x_{2}, x_{1}\right)$. Qualitatively, the trajectory $\tilde{r}(x)$ is related to $r(x)$ in the same way as $\hat{r}(x)$ is in Fig. 9. The differential equation in (A.1) is separable and we may integrate it directly,

$$
\int_{r_{2}}^{\tilde{r}} \frac{\mathrm{d} \tilde{r}}{\left(s\left(P-t_{2}\right)+\tilde{r}^{2}\right) \tilde{r}}=-\int_{x_{2}}^{x} \frac{\mathrm{d} x}{x}, \quad \frac{1}{2 s\left(P-t_{2}\right)} \ln \left(\frac{\tilde{r}^{2}\left(s\left(P-t_{2}\right)+r_{2}^{2}\right)}{r_{2}^{2}\left(s\left(P-t_{2}\right)+\tilde{r}^{2}\right)}\right)=-\ln \left(\frac{x}{x_{2}}\right),
$$

which re-arranges to give

$$
\tilde{r}^{2}=\frac{s\left(P-t_{2}\right)}{\left(\left(s\left(P-t_{2}\right) / r_{2}^{2}\right)+1\right)\left(x / x_{2}\right)^{2 s\left(P-t_{2}\right)}-1} .
$$

Since $\tilde{r}^{2}\left(x_{1}\right)<r_{1}^{2}$ it then follows from Eq. (A.2) that

$$
F:=r_{1}^{2}-\frac{s\left(P-t_{2}\right)}{\left(\left(s\left(P-t_{2}\right) / r_{2}^{2}\right)+1\right) \mathrm{e}^{2 s P\left(P-t_{2}\right)}-1}>0,
$$

where we have used the fact that $x_{1} / x_{2}=\mathrm{e}^{P}$. We now write the traversal time, $P$, as a Taylor series in $s$,

$$
P=\sum_{n=0}^{\infty} A_{n} s^{n}
$$

substitute this expression into $F$ (the left side of the inequality (A.3)), expand the resulting expression for $F$ as a Taylor series in $s$ and solve for the coefficients $A_{n}$ which cause $F$ to vanish. To determine whether the resulting Taylor series for $P$ is an upper or lower bound, we must determine whether an increase or decrease in $P$ will give $F>0$ for small $s$. Since, as we have already noted, system (A.1) approaches system (20) as $s \rightarrow 0$, we may use the results from the analysis of (20) for $s=0$. In particular, from inequality (21) we get

$$
\lim _{s \rightarrow 0} F=r_{1}^{2}-\frac{1}{\left(1 / r_{2}^{2}\right)+2 P},
$$

so that $F$ increases as $P$ increases and thus the Taylor series is a lower bound for $P$. The first three terms of this series are given in Table 2(A).

An upper bound for $P$ may be obtained by substituting the expression for $\tilde{r}^{2}$ given by (A.2) into $K$ (14). Since $\tilde{r}(x)<r(x)$ on $\left(x_{2}, x_{1}\right)$, we have

$$
K>\int_{x_{2}}^{x_{1}} \frac{\tilde{r}^{2}}{x} \mathrm{~d} x .
$$

Using the expression for $\hat{r}^{2}$ (A.2) and the antiderivative

$$
\int \frac{\mathrm{d} z}{z\left(a z^{p}+b\right)}=\frac{1}{p b} \ln \left(\frac{z^{p}}{a z^{p}+b}\right),
$$


Table A.1

Taylor series in $s$ for bounds on travel time

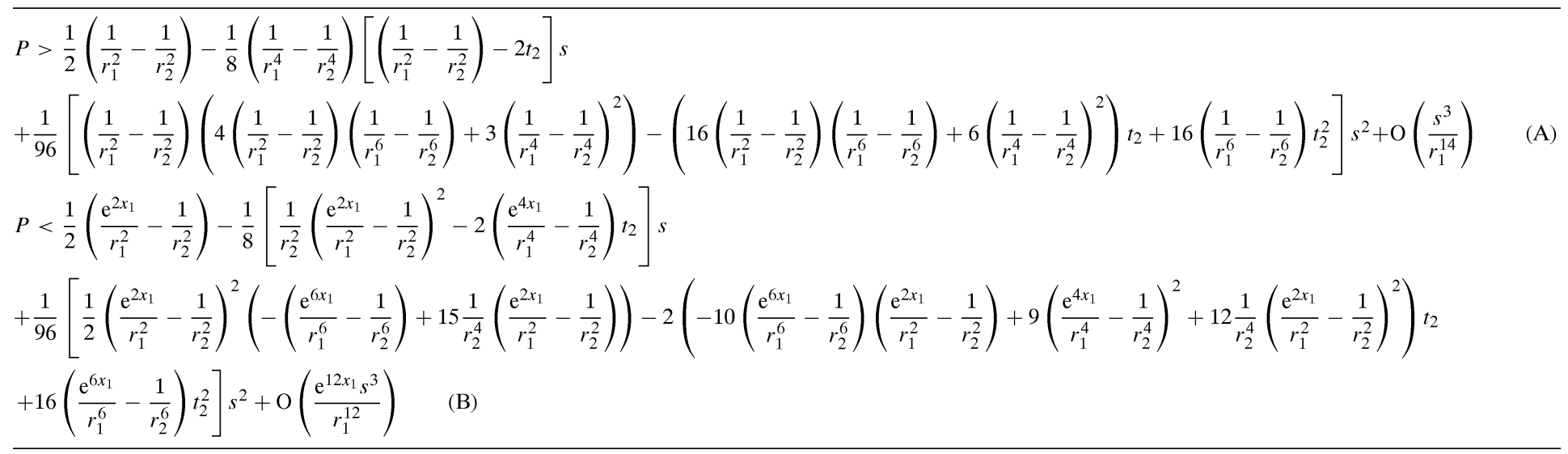


the right side of inequality (A.5) integrates giving

$$
K>\frac{1}{2}\left(\ln \left(\frac{\left(\left(s\left(P-t_{2}\right) / r_{2}^{2}\right)+1\right)\left(x_{1} / x_{2}\right)^{2 s\left(P-t_{2}\right)}-1}{\left(s\left(P-t_{2}\right) / r_{2}^{2}\right)}\right)-\ln \left(\left(\frac{x_{1}}{x_{2}}\right)^{2 s\left(P-t_{2}\right)}\right)\right) .
$$

Again, using $x_{1} / x_{2}=\mathrm{e}^{P}$, we get

$$
K>\frac{1}{2} \ln \left(\frac{\mathrm{e}^{2 s P\left(P-t_{2}\right)}\left(\left(s\left(P-t_{2}\right) / r_{2}^{2}\right)+1\right)-1}{\left(s\left(P-t_{2}\right) / r_{2}^{2}\right)}\right)-s P\left(P-t_{2}\right),
$$

while from Eq. (15), since $\mathrm{e}^{-P}<1$, we have

$$
K<\ln \left(\frac{r_{2}}{r_{1}}\right)+x_{1}+s t_{2} P-\frac{s}{2} P^{2} .
$$

Combining the above two inequalities, multiplying by 2 and exponentiating gives

$$
\left(\frac{\mathrm{e}^{2 s P\left(P-t_{2}\right)}\left(\left(s\left(P-t_{2}\right) / r_{2}^{2}\right)+1\right)-1}{\left(s\left(P-t_{2}\right) / r_{2}^{2}\right)}\right) \mathrm{e}^{-2 s P\left(P-t_{2}\right)}<\left(\frac{r_{2}}{r_{1}}\right)^{2} \mathrm{e}^{2 x_{1}} \mathrm{e}^{2 s t_{2} P-s P^{2}},
$$

which simplifies to

$$
G:=\frac{s\left(P-t_{2}\right)}{r_{1}^{2}} \mathrm{e}^{2 x_{1}} \mathrm{e}^{-s P\left(P-2 t_{2}\right)}-\frac{s\left(P-t_{2}\right)}{r_{2}^{2}}-1+\mathrm{e}^{-2 s P\left(P-t_{2}\right)}>0 .
$$

We again write $P$ as a Taylor series in $s$ (A.4), substitute this expression into $G$, expand the resulting expression for $G$ as a Taylor series in $s$ and solve for the coefficients $A_{n}$ which cause $G$ to vanish. As before, to determine whether the resulting Taylor series for $P$ is an upper or lower bound, we must determine whether an increase or decrease in $P$ will give $G>0$ for small $s$. To order one in $s, G$ is given by

$$
G=s\left(P-t_{2}\right)\left(\frac{1}{r_{1}^{2}} \mathrm{e}^{2 x_{1}}-\frac{1}{r_{2}^{2}}-2 P\right)+\mathrm{O}\left(s^{2}\right)
$$

Since the terms $s\left(P-t_{2}\right)$ and $\left(1 / r_{1}^{2}\right) \mathrm{e}^{2 x_{1}}-\left(1 / r_{2}^{2}\right)$ are both necessarily positive, it follows that for small $s, G$ goes from positive to negative as $P$ increases. Thus the Taylor series for $P$ represents an upper bound, the first three terms of which are given in Table 2(B).

In the lower bound for $P$, Table 2A, the order $s^{n}$ term has a factor of $r_{1}^{-(4 n+2)}$, while the upper bound, Table 2B, has the same factor for the $n$-even terms and a factor of $r_{1}^{-4 n}$ for the $n$-odd terms. Thus, both series are convergent provided $s<r_{1}^{4+\epsilon}$, where $\epsilon$ is any positive number; and all terms of order $s^{n}$ with $n>(2 / \epsilon)$ will vanish as $r_{1} \rightarrow 0$. For example, the first three terms, as given above, provide a good approximation to the asymptotic limit if $s<r_{1}^{4+\epsilon_{1}}$, where $\epsilon_{1}>1 / 2$. Note also that as $s \rightarrow 0$, these estimates approach those obtained for the frozen system.

\section{References}

[1] L. Belyakov, A case of the generation of periodic motion with homoclinic curves, Math. Notes 15 (1974) 336-341.

[2] M. Bosch, C. Simo, Attractors in a Silnikov-Hopf scenario and a related one-dimensional map, Phys. D 62 (1993) $217-229$.

[3] B. Deng, K. Sakamoto, Silnikov-Hopf bifurcations, J. Differential Equations 119 (1995) 1-23.

[4] M. Frankel, V. Rotyburd, A free boundary problem modeling thermal instabilities: stability and bifurcation, J. Dynamics Differential Equations 6 (1994) 447-486. 
[5] J. Guckenheimer, R. Harris-Warrick, J. Peck, A. Willms, Bifurcation, bursting and spike-frequency adaptation, J. Comput. Neurosci. 4 (1997) 257-277.

[6] J. Guckenheimer, P. Holmes, Nonlinear Oscillations, Dynamical Systems and Bifurcation of Vector Fields, Springer, New York, 1983.

[7] P. Hirsch, E. Knobloch, Silnikov-Hopf bifurcation, Phys. D 62 (1993) 202-216.

[8] S. Newhouse, The abundance of wild hyperbolic sets and non-smooth stable sets for diffeomorphisms, Publ. Math. IHES 50 (1979) $101-151$.

[9] J. Rinzel, B. Ermentrout, Analysis of neural excitability and oscillations, in: C. Koch, I. Segev (Eds.), Methods of Neural Modeling: From Synapses to Networks, MIT Press, Cambridge, MA, 1989, 135-169. 